\title{
Competency assessment for trainers
}

We, as trainers, are often expected to 'teach to the test' because a test is perceived as the main assessment of the competency of investigators and animal care providers to work with animals in research, testing and teaching. But, as Hansen and Sørensen point out in a recent paper on strategies for assessing competence in lab animal science courses (Lab Anim. (NY) 43, 359-363; 2014), "[a] single examination strategy can never entirely assess the various competencies of individual students or evaluate all learning outcomes." Trainers in lab animal science should therefore diversify their assessment strategies instead of relying solely on traditional tools like multiple-choice questions. Let me elaborate on my thoughts.

Hansen and Sørensen describe the redesign of testing in courses offered by the Federation of European Laboratory Animal Science Associations (FELASA), which places "a greater emphasis on learning outcomes" and "for the first time, sets standards for the assessment of learning outcomes." They also mention knowledge gained, practical experience, deep learning and critical thinking - the same elements currently being deployed in US public schools in the Common Core State Standards (http://www.corestandards.org/). This will make sense to lab animal trainers around the world: learning procedures and memorizing facts are important, but the best competency is being able to apply knowledge appropriately in the vivarium. Hansen and Sørensen also identify several challenges to such assessments, such as ensuring consistency across broad geographical areas, evaluating very specific practical skills and implementing tests that reliably assess the anticipated learning outcomes.

I recommend that trainers read Hansen and Sørensen's article and other materials about evaluations, such as Popham's Everything School Leaders Need to Know about Assessment (Corwin/Sage, Thousand Oaks, CA, 2010) or Fenwick and Parsons' The Art of Evaluation: A Handbook for Educators and Trainers (Thompson Educational Publishing, Inc., Toronto, Canada, 2000). Popham, for example, describes the concept of formative assessment that happens along the way during training: "Formative assessment is a planned process in which assessmentelicited evidence of students' status is used by teachers to adjust their ongoing instructional procedures or by students to adjust their current learning tactics." This process might be most applicable to training that occurs over a long period of time, allowing for multiple formative tests. But it can also be incorporated into shorter, finite training sessions, for example, by administering quizzes before and after the session to measure knowledge gained. Trainers might need to use their creativity in order to develop such assessments.

Help comes in the form of two other recently published papers (J. Am. Assoc. Lab. Anim. Sci. 52, 711-716; 2013 and Lab. Anim. Sci. Profess. 1, 24-25; 2013) that discuss using rubrics or scales of ability ranging from none to partial to competent. I found them useful for initiating assessments into a training program.

The American Association for Laboratory Animal Science (AALAS) long ago applied the science of psychometrics to its technician certification examinations as a better measure of competency. While I was on the Animal Technician Certification Board, members reviewed the relevance of every question to the conduct of lab animal technology, set passing scores on exams to assure minimum competency and evaluated the statistical performance of both individual questions and whole tests when given (on a quarterly basis). Some readers will remember that the practical portion of the examination included slides. As a trainer, I encourage the incorporation of images and reference sources into the computerized examinations we have today. I believe that allowing examinees to look up a fact or figure and use it to answer a question (e.g., determining how many mice can be housed in a cage after calculating the floor space) provides a better assessment of their ability to apply critical thinking skills than does expecting them to memorize tables of information. Similarly, asking examinees to identify an organ that is located in the abdominal cavity by pointing to it in a photo or diagram, instead of choosing it from a list of options, might better assess anatomical knowledge.

The Canadian Association for Laboratory Animal Science (CALAS) holds examinations for Registered Master Laboratory Animal Technician (RMLAT) certification during its annual symposia. This strategy is successful probably because only a few candidates are tested and they are all in one place, but it also has several drawbacks: it is very resource-intensive for the committee, it happens only once per year and it needs to address concerns about subjectivity in responses. Both FELASA and CALAS (for the RMLAT certification) utilize long essay questions and papers in their assessments of a candidate. I am not convinced these tools would be practical for the AALAS technician certification program, but I believe they could be incorporated into its Certified Manager of an Animal Resource program.

In sum, I argue that competency in lab animal science must be assessed in ways other than multiple-choice examinations, as Hansen and Sørensen wrote. As trainers, we can be a part of the innovation. 